CANCER

\section{Cause and Cure}

\section{from our Medical Biochemistry Correspondent}

ONE of the most striking differences between tumour cells and normal mammalian cells is the abnormality of the surface of the tumour cells. Features of the lipid components of the tumour cell might be the cause of the change in the cell surface, for lipids, and particularly phospholipids, are important components of biological membranes. Wood and Snyder (Arch. Biochem. Biophys., 131, 478; 1969) have found that the specificity of enzymes synthesizing triglycerides and phosphoglycerides in the cells of the Ehrlich ascites tumour seems to be different from those synthesizing these compounds in rat liver (Wood and Harlow, Arch. Biochem. Biophys., 131, 495; 1969).

For each tissue, they separated the lipids into the neutral triglycerides and the different phosphoglyceride fractions. They then removed the fatty acids from the 1, 2 and 3 positions of the glyceride molecule, and separated the fatty acid mixtures to determine the proportions of different fatty acids esterified at each position. They then calculated the molecular weight distribution which would be expected within each class if the fatty acids found were distributed randomly in the glyceride molecules. This they compared with their own experimental determination of the distribution of molecular weights in each class of glyceride (determined by gas-liquid chromatographic separation). For the ascites tumour the actual distribution agreed very well with the calculated random distribution for each class so that in this tissue the synthesis of glycerides appeared to proceed in a completely random manner. In the rat liver, however, the relative proportions of glycerides of different sizes differed considerably from that predicted by the random hypothesis, showing that there must be preferential pairing of certain fatty acids in some of the molecules. This difference could have two explanations. The tumour cell enzymes which synthesize glycerides may be less specific in their requirements for diglyceride substrates than the rat liver enzymes, or the liver may contain. acyl CoA: lysophosphatide acyl transferase enzymes which are not present in the malignant cells. Whatever the explanation, this detailed structural study has shown that there are differences in tumour glycerides which might affect the cell mombrane.

Biochemists are interested in the control of cell division in cancer cells as well as in abnormalities of metabolism. Vince and Ward have described a new approach to cancer chemotherapy in a recent issue of Biochemical and Biophysical Research Communications $(35,593 ; 1969)$. The carcinostatic properties of $\alpha$-ketoaldehydes suggested that cells might control their rate of division by regulating the concentration of ketoaldehydes such as methylglyoxal. This substance is converted to lactic acid by glyoxalase, which requires glutathione as a coenzyme. And so Vince and. Ward thought that an inhibitor of glyoxalase might maintain the concentration of methylglyoxal in the cell and thus stop division. They therefore looked for some effective inhibitors of glyoxalase. Although S-methylglutathione was known to inhibit glyoxalase, they found that glutathione derivatives with longer side-chains are much more effective inhibitors. They think that a hydrophobic region of the enzyme binds the alkyl side-chain so that the competitive inhibition increases as the side-chain increases from methyl to n-octyl (derivatives with longer side-chains were too insoluble to be useful). Vince and Ward have thus produced glyoxalase inhibitors which are 435 times as active as S-methylglutathione, and the results of their investigation of these as potential anti-cancer drugs will be awaited with interest.

\section{GENDER IDENTITY \\ The Desire to be Different}

\section{from our Special Correspondent}

THE first international symposium on gender identity was held behind closed doors in London last week, but on July 27 some of the participants emerged to talk in public. The problem they had been discussing is that of the transsexual, who has an overwhelming wish to belong to the opposite sex although there are no doubts as to the anatomical sex. The feelings involved are so strong for these people that the only treatment they will accept is often an operation for change of sex.

Because transsexuals do not usually want to be cured of their longings, psychotherapy seems to have little to offer. Treatment with hormones sometimes helps. In male transsexuals, who constitute the greater proportion of cases, oestrogens produce partial and temporary "chemical castration" which helps to alleviate the desire to be rid of the sex organs. John Randell (Char. ing Cross Hospital, London) explained that in cases when the desire to change sex is very strong, it seems reasonable to carry out surgery, after which there is often an improvement in the social adjustment of the patient. For males, the operation involves the removal of penis and testes (sometimes an artificial vagina is given); for females the operation is a hysterectomy.

John Money (Johns Hopkins Hospital) agreed that if cases are carefully selected, the patient's situation is undoubtedly improved by surgery. Since 1953, 1,000 operations have been performed in various parts of the world. Peter Scott (Bethlem Royal and Maudsley Hospitals) considers that psychotherapy has an important part to play. He fecls that some men wish to become women so that they can have a position in society which involves less anxiety and tension. Scott feels that these men can be helped by showing them how to occupy a less mentally strenuous niche in society, not necessarily by becoming women.

The causes of transsexualism are largely a mystcry. No genetic cause has been found, although the distinctly feminine build of many male transsexuals suggests such a cause. Recent work has pointed to a neuroendocrine cause. Among others S. Levine (Sci. Amer., 214, 84; 1966; Science, 142, 1585; 1966) described a hypothalamic centre which is basically female in all human foetuses. At a critical time in development this has to be masculinized in the male by androgens from the tiny foctal testes. If this does not occur the centre remains female and may well determine the sexual behaviour of the future child.

Childhood conditioning also seems to be important. Richard Green (University of California, Los Angoles) described cases of transsexual children brought up in 